\title{
INFLUENCE OF AUXIN ON BIOMASS PRODUCTION AND WITHANOLIDE ACCUMULATION IN ADVENTITIOUS ROOT CULTURE OF INDIAN RENNET: WITHANIA COAGULANS
}

\author{
PARAMESWARI MURUGESAN, KALAISELVI SENTHIL*
}

Department of Biochemistry, Biotechnology and Bioinformatics, Avinashilingam Institute for Home Science and Higher Education for Women, Coimbatore, Tamil Nadu, India. Email: kalaiselvi_bc@avinuty.ac.in

Received: 28 January 2017, Revised and Accepted: 13 April 2017

\section{ABSTRACT}

Objective: Withanolides are the biologically active, principle compound present in Withania coagulans, which is having a high medicinal value and possesses potent therapeutic activity. The present study was attempted with an objective to investigate a biomass growth and withanolide production in in vitro root tissues of $W$. coagulans.

Methods: High-performance thin layer chromatography (HPTLC) often serves as a method for quantification of major withanolides. In the present study, methanolic withanolide extract of in vitro cultured W. coagulans root tissue were carried out using HPTLC. The HPTLC analysis was performed using precoated silica gel aluminum plate $(20 \mathrm{~cm} \times 20 \mathrm{~cm}) 60 \mathrm{~F} 254$ (E.MERCK, Germany) with mobile phase toluene: ethyl acetate: formic acid (5:5:1).

Results: The optimization of different combination and concentration of plant growth regulators (indole-3-butyric acid [IBA] and indole-acetic acid) was used to stimulate the biomass growth and withanolide production. The maximum biomass growth $(7.48 \pm 0.25 \mathrm{~g} / \mathrm{dL}) \mathrm{was}$ observed in medium with $4.93 \mu \mathrm{M}^{-1}$ IBA. The higher amount of withanolide A (204.98 $\left.\pm 0.87 \mu \mathrm{g} / \mathrm{L} \mathrm{DW}\right)$ and withaferin A $(227.15 \pm 0.57 \mu \mathrm{g} / \mathrm{L} \mathrm{DW})$ accumulation was recorded in culture grown on half Murashiga-Skoog media supplemented with $4.93 \mu \mathrm{M}^{-1}$ and $2.46 \mu \mathrm{M}^{-1} \mathrm{IBA}_{\text {. }}$

Conclusion: We concluded that in vitro-cultured system could provide unique opportunities for large-scale production of pharmaceutically important compound than field grown plants.

Keywords: Adventitious root culture, High-performance thin layer chromatography, Withania coagulans, Withanolides.

(C) 2017 The Authors. Published by Innovare Academic Sciences Pvt Ltd. This is an open access article under the CC BY license (http://creativecommons. org/licenses/by/4. 0/) DOI: http://dx.doi.org/10.22159/ajpcr.2017.v10i7.17342

\section{INTRODUCTION}

The genus Withania (Family: Solanaceae) is a highly acclaimed genus of medicinal plants in the Indian ayurvedic system of medicine because of its magnificent pharmaceutical and nutraceutical properties [1]. Withania is a small genus of shrubs which are distributed in the east of the Mediterranean region and extend to South Asia. Among the 23 known species of Withania, only two, Withania somnifera and Withania coagulans, are economically considerable and widely cultivated [2,3]. W. coagulans Dunal is one of the vital medicinal plants. It is commercially important for its milk coagulating properties [4] and is well known in the indigenous system of medicine for the treatment of ulcers, dyspepsia, rheumatism, dropsy, consumption, and senile debility. It has received much attention in recent years due to the presence of a large number of steroidal alkaloids and lactones known as withanolides [5].

The major biochemical constituent of this plant is withanolides. They are a group of naturally occurring C-28 steroidal lactones built on an intact or rearranged ergostane agenda, in which C-22 and C-26 are appropriately oxidized to form a 6-membered lactone ring [6]. Biogenetic transformations of the steroidal skeleton and side chain have diversified the structure of withanolides. It have attracted attention due to their extensive range of biological activities, including antitumor, anti-inflammatory, antimicrobial, cytotoxic, immunomodulating, and cancer chemopreventive activities [7,8]. Withaferin A, withanolide A, and withanone are the major withanolides present in W. somnifera and W. coagulans.

The in vitro propagation technique may be the greatest solution for its rapid multiplication and reestablishment in nature [9]. It also provides an alternative to field grown plant harvesting for the production of therapeutically valuable compounds [10] and is reported that the withanolide contents of the in vitro hairy root cultures of $W$. coagulans were higher than in the root of the plant [11]. Recently, phytohormones specifically auxin plays an essential role in regulating root development and it has been shown to be intimately involved in the production of adventitious root growth and withanolide accumulation in $W$. somnifera [12]. Auxin, indole-acetic acid (IAA) was shown to be involved in the rooting process by Thimann and Went as far back as 1934, and a second "synthetic" auxin indole-3-butyric acid (IBA) also promoted rooting [13]. Adventitious root formation has many practical implications in horticulture and agronomy and there is a lot of commercial interest because of the many plant species that are difficult to root $[14,15]$. Therefore, there is a need to develop an efficient protocol for the induction of in vitro adventitious roots of $W$. coagulans.

Root cultures can be used as standard experimental system in studies of carbohydrate metabolism, mineral nutrient requirements, essentiality of vitamins and other growth regulators, differentiation of the root apex and gravitropism. The advantage of using root cultures is that they grow rapidly, relatively easy to prepare and maintain, show a low level of variability and can be easily cloned to produce a large supply of experimental tissue [16]. Further improvement of biomass and withanolide accumulation can also be achieved by medium containing manipulation of the exogenous supplement of auxins. IBA and IAA were responsible for the adventitious root culture of $W$. somnifera and also the combination of IBA and IAA was promoted adventitious root induction in various plant materials [17]. Internally, macronutrients such as nitrogen play an important role not only in the growth of tissue cell lines but also in the production of biomass and withanolide in root suspension cultures [18].

To the best of our knowledge, there have been no reports on the effect of auxin combination on the biomass and withanolide accumulation in root 
suspension cultures of $W$. coagulans. Therefore, we have investigated the effects of different auxin combination on root suspension culture of $W$. coagulans in terms of biomass and withanolide accumulation.

\section{METHODS}

\section{Plant materials}

The seeds of $W$. coagulans were obtained from Banaras Hindu University, Varanasi. Surface-sterilized seeds were then germinated in vitro in Murashige-Skoog (MS) media supplemented with $2 \%$ sucrose and seedlings produced were maintained on MS basal medium.

\section{In vitro adventitious root induction}

The young or mature leaves from 2 months old seedlings were used as explant for the present study. The leaves were cut into $0.5 \mathrm{~cm}^{2}$ and inoculated on MS [19] medium with $3 \%(\mathrm{w} / \mathrm{v})$ sucrose, $0.8 \%(\mathrm{w} / \mathrm{v})$ agar, and supplemented with concentration of auxins such as $\left(5.71 \mu \mathrm{M}^{-1}\right)$ IAA and $\left(19.7 \mu \mathrm{M}^{-1}\right)$ IBA were added. The cultures were kept under 16-hrs photoperiod $\left(40 \mu \mathrm{mol} / \mathrm{m} \mathrm{s}^{-1}\right)$ provided by $40 \mathrm{~W}$ fluorescent lamps (Philips, Kolkata, India) at $25 \pm 2^{\circ} \mathrm{C}$. The growth of adventitious root was recorded at weekly intervals. The roots were transferred into half strength MS suspension medium for further studies [17].

Effect of auxin combination on adventitious root growth and withanolide production

After a period of 30 days, the root tips (about 25-30) from root induction medium were cut under sterile condition and transferred into half strength MS liquid medium containing different concentration of IBA and IAA and also in combination of both the IBA and IAA along with 3\% sucrose (Table 1). All the culture vessels were kept under continuous agitation at $110 \mathrm{rpm}$ in an orbital shaker (Orbritek, Scigenics, and Chennai, India) and incubated at $25 \pm 2^{\circ} \mathrm{C}$, with a $16 \mathrm{hrs}$ photoperiod. The biomass growth and withanolides production were measured after 4 weeks of culture. The roots were harvested and their wet and dry weight were recorded after that the roots were dried to a constant weight at $60^{\circ} \mathrm{C}$ for $24 \mathrm{hrs}$.

GI $=\frac{\text { Fresh weight of harvested biomass }- \text { fresh weight of the inoculums }}{}$ Fresh weight of the inoculums

\section{Extraction of secondary metabolites}

The dried root samples were ground thoroughly using mortar pestle and root powder was obtained. Initially, $1 \mathrm{~g}$ of root powder was weighed and treated with $1 \mathrm{ml}$ ammonia for 20 minutes at room temperature, followed by sonication for 20 minutes with $50 \mathrm{ml}$ of methanol and placed in a shaker for $2 \mathrm{hrs}$ at $150 \mathrm{rpm}$ at $22^{\circ} \mathrm{C}$. At the end of $2 \mathrm{hrs}$, the extract was filtered using whatmann no.1 filter paper and the residues were again treated with $50 \mathrm{ml}$ methanol. This step was repeated four times to obtain $200 \mathrm{ml}$ of the extract. The extract was then concentrated by evaporation using flash evaporator maintained at $45^{\circ} \mathrm{C}$ and $150 \mathrm{rpm}$. After complete evaporation, the residue was dissolved using highperformance liquid chromatography grade methanol [20].

\section{Quantification of major withanolides}

High-performance thin layer chromatography (HPTLC) was performed on precoated silica gel aluminum plate $(20 \mathrm{~cm} \times 20 \mathrm{~cm})$ 60F254 (E.MERCK, Germany). The methanolic extract of $W$. coagulans was loaded to the plates as $6 \mathrm{~mm}$ bands, under a stream of nitrogen using the Camag (Switzerland) Linomat V semiautomatic sample applicator fitted with a $100 \mu$ l of Hamilton HPTLC syringe. The HPTLC plates were developed up to $80 \mathrm{~mm}$ using the mobile phase toluene: ethyl acetate: Formic acid in the ratio of 5:5:1, respectively, in a Camag Twin trough glass tank. It was presaturated with the mobile phase solvents for 30 minutes at room temperature $\left(25 \pm 2^{\circ} \mathrm{C}\right)$. The developed plate was air dried and the image was captured at $245 \mathrm{~nm}$ and $366 \mathrm{~nm}$. Densitometric scanning was performed at $235 \mathrm{~nm}$ for withanolide A and $530 \mathrm{~nm}$ for withaferin A using Camag TLC scanner III controlled by Camag CATS 4 integration software. The Rf values of the resolved spots were noted. The peak areas were evaluated using linear regression [21].
Table 1: Hormonal combination

\begin{tabular}{lll}
\hline Treatments & IBA $\left(\boldsymbol{\mu \mathbf { M } ^ { - 1 } )}\right.$ & IAA $\left(\boldsymbol{\mu \mathbf { M } ^ { - 1 } )}\right.$ \\
\hline T0 (MS0) & 0 & 0 \\
T1 & 1.23 & 0 \\
T2 & 2.46 & 0 \\
T3 & 4.93 & 0 \\
T4 & 0 & 0.35 \\
T5 & 0 & 0.71 \\
T6 & 0 & 1.43 \\
T7 & 4.93 & 0.35 \\
T8 & 4.93 & 0.71 \\
T9 & 4.93 & 1.43 \\
T10 & 1.23 & 1.43 \\
T11 & 2.46 & 1.43 \\
\hline
\end{tabular}

IBA: Indole-3-butyric acid, IAA: Indole-acetic acid, T0 - Control, T1 - Half strength liquid MS medium supplemented with $0.25 \mathrm{mg} / \mathrm{L}$ IAA, T2-0.5 mg/L IAA, T3-1 mg/L IAA, T4-0.25 mg/L IBA, T5-0.5 mg/L IBA, T6-1 mg/L IBA T7-0.25 mg/L IAA and $1 \mathrm{mg} / \mathrm{L}$ IBA, T8-0.5 mg/L IAA and $1 \mathrm{mg} / \mathrm{L} \mathrm{IBA,} \mathrm{T9-1}$ $\mathrm{mg} / \mathrm{L}$ IAA and IBA, T10-0.25 mg/L IBA and $1 \mathrm{mg} / \mathrm{L}$ IAA, T11-0.5 mg/L IBA and $1 \mathrm{mg} / \mathrm{L} \mathrm{IAA}$

The amount of withaferin $\mathrm{A}$ and withanolide $\mathrm{A}$ in the samples was quantified using peak area. The plates were derivatized using anisaldehyde sulfuric acid ( $85 \mathrm{~mL}$ methanol: $10 \mathrm{~mL}$ glacial acetic acid: $5 \mathrm{~mL}$ sulfuric acid: $0.5 \mathrm{~mL}$ anisaldehyde) reagent and placed in hot air oven for 2 minutes at $110^{\circ} \mathrm{C}$ for detection of spots.

\section{RESULTS AND DISCUSSION}

Influence of auxins on lateral root growth and withanolides production

Plant growth regulators (PGRs) are organic substance which is used to regulate the plant growth and also modify the plant physiological processes. They may act as either biostimulants (or) bioinhibitors, act inside plant cells to stimulate (or) inhibit specific enzymes or enzyme system, and help regulate plant metabolism [22]. The early characterization of auxins as "root-forming substance of plants" due to established a long-standing relationship between this group of small molecules and lateral root development [23,24]. The aerial portion of the plant body, a series of iterative modules produces the overall root architecture; the root which is established during embryogenesis and gives rise to new lateral roots branches in a continuous, indeterminate manner. Proof from many research has been indicate the vital role of auxins in orchestrating the final root architecture. The major role of auxins as a component of endogenous developmental programs as well as in mediating environmental stimuli to shape the final root architecture remains at the heart of several active research programs [25].

Lateral roots are initiated from root pericycle cells adjacent to the protoxylem poles of the parent root [26]. Although there remains a paucity of data that defines the molecular machinery responsible for generating a lateral root, current observations point out that the process progresses through at least four recognizable phases: Priming, initiation, patterning, and emergence $[27,28]$. Each of these phases is controlled or at least influenced by auxin [25]. Since they are control the growth of stem, roots, fruits, and convert stem into flowers. It acts to inhibit the growth of buds and also promote the biomass growth and secondary metabolites production [29].

Among the dynamic auxins, IBA and IAA are the most frequently used Plant Growth Regulator because it is not rapidly degraded by the plant and is not translocated from the site of application. However, the high concentrations of auxins are toxic to plants. They are most toxic to dicots than monocots. Because of this property, synthetic auxin herbicides including 2,4-D and 2,4,5-T have been developed and used for weed control. In this regard, lower concentration of auxins is highly peripheral for lateral root growth and plant-derived secondary metabolites production [30].

Exogenous supplement of auxin is an important factor for adventitious root formation. However, endogenous auxins may also play a role in 


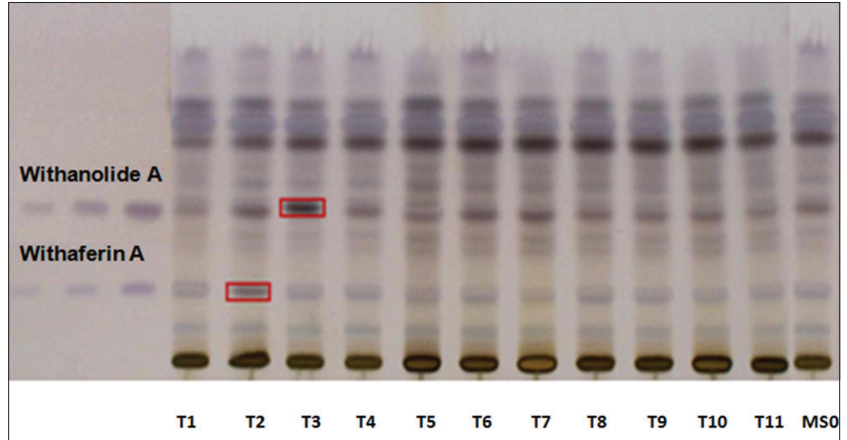

Fig. 1: Analysis of Withanolide A and Withaferin A on auxin treated in vitro roots of $W$. coagulans. Lane 1-3: Standards withanolide

A and withaferin A, T1 - Half strength liquid Murashigae-

Skoog medium supplemented with $0.25 \mathrm{mg} / \mathrm{L}$ Indole-acetic acid (IAA), T2 - $0.5 \mathrm{mg} / \mathrm{L} \mathrm{IAA,} \mathrm{T3} \mathrm{-} 1 \mathrm{mg} / \mathrm{L}$ IAA, T4 - $0.25 \mathrm{mg} / \mathrm{L}$ Indole-3-butyric acid (IBA), T5 - $0.5 \mathrm{mg} / \mathrm{L}$ IBA, T6 - $1 \mathrm{mg} / \mathrm{L}$ IBA T7 - $0.25 \mathrm{mg} / \mathrm{L}$ IAA and $1 \mathrm{mg} / \mathrm{L} \mathrm{IBA,} \mathrm{T8} \mathrm{-} 0.5 \mathrm{mg} / \mathrm{L}$ IAA and $1 \mathrm{mg} / \mathrm{L}$ IBA, T9 - $1 \mathrm{mg} / \mathrm{L}$ IAA and IBA, T10 - $0.25 \mathrm{mg} / \mathrm{L}$ IBA and $1 \mathrm{mg} / \mathrm{L}$ IAA, T11 - $0.5 \mathrm{mg} / \mathrm{L}$ IBA and $1 \mathrm{mg} / \mathrm{L} \mathrm{IAA,} \mathrm{MSO} \mathrm{(Control)}$

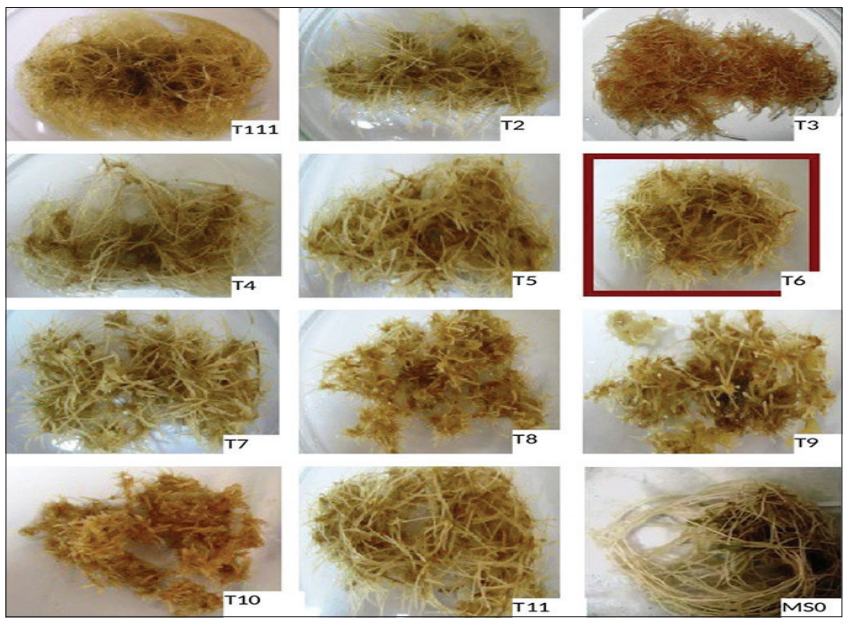

Fig. 2: Lateral root branch formation in different auxin concentrations

the rooting process. Therefore, medium with altered auxin levels was investigated for their ability to form adventitious roots mass and plant metabolites production [31]. To our knowledge, well clearly indicated that type and concentration of auxins strongly influence the formation of lateral root, thereby increasing the growth index was represented in Table 2 and Figs. 1 and 2.

Lateral roots were initiated within 2 weeks of culture, after exposure to the auxins; whereas without auxins, there was no lateral roots formation. The lateral root elongated further and become a bristle appearance. The root supplemented with IBA at $4.93 \mu \mathrm{M}^{-1}$ concentration invoked better biomass growth than compared to other combinations and concentrations. The HPTLC analysis of in vitro root samples of $W$. coagulans was performed using the solvent system tolene: ethyl acetate:formic acid (5:5:2) to check the accumulation of withanolide $\mathrm{A}$ and withaferin A. The result revealed that among the different combination and concentrations of auxins (IBA and IAA).

IBA at high concentration $4.93 \mu \mathrm{M}^{-1}$ individually stimulates the lateral root branches $(7.48 \pm 0.25 \mathrm{~g} / \mathrm{dL})$ and withanolide A $(204.98 \pm 0.87 \mu \mathrm{g} / \mathrm{L} \mathrm{DW})$ production after 30 days of root suspension culture (Table 2, Graph 1, and Fig. 1). Whereas medium with $2.46 \mu \mathrm{M}^{-1}$ IBA favors relatively high accumulation of withaferin A $(227.15 \pm 0.57)$ (Graph 2).

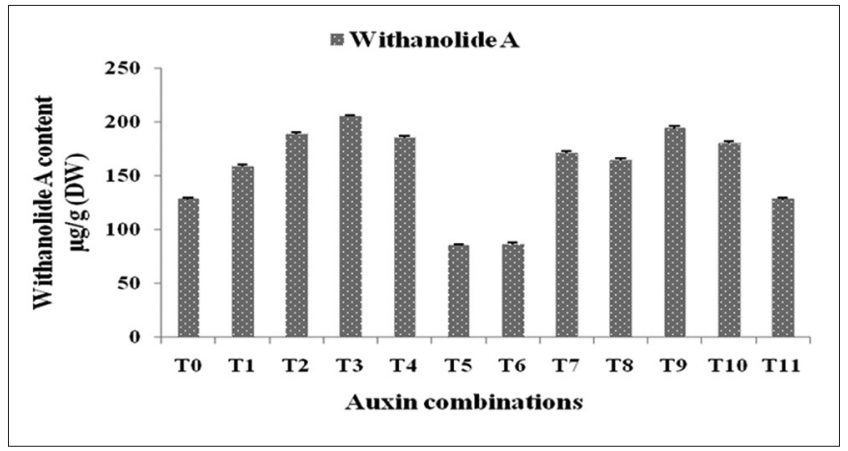

Graph 1: Influence of auxins on withanolide A accumulation after 30 days of root suspension culture

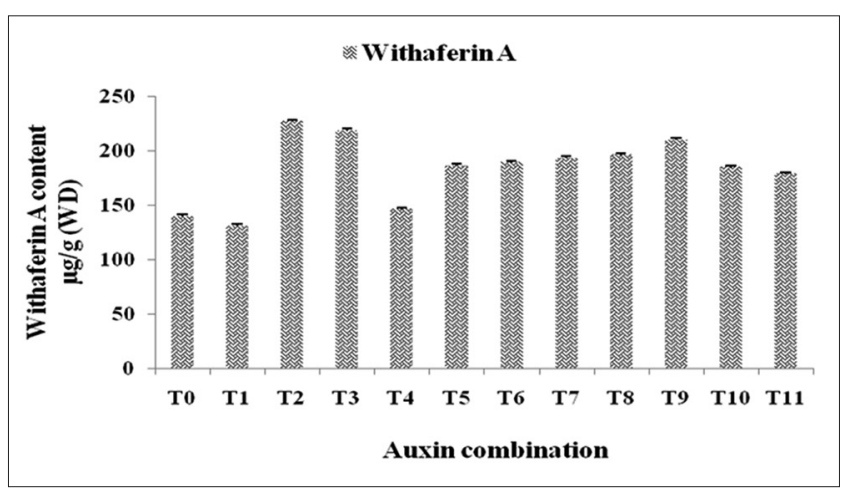

Graph 2: Influence of auxins on withaferin A accumulation after 30 days of root suspension culture

Table 2: Effect of auxins on growth index

\begin{tabular}{lll}
\hline Different auxin treatments & $\begin{array}{l}\text { Harvested fresh } \\
\text { weight }(\mathrm{g} / \mathbf{d L})\end{array}$ & Growth index \\
\hline MS0 & $2.18 \pm 0.17$ & 9.9 \\
T1 & $4.38 \pm 0.41$ & 20.9 \\
T2 & $5.30 \pm 0.37$ & 25.5 \\
T3 & $7.48 \pm 0.25$ & 36.4 \\
T4 & $3.37 \pm 0.43$ & 15.85 \\
T5 & $6.45 \pm 0.29$ & 31.25 \\
T6 & $6.85 \pm 0.84$ & 33.25 \\
T7 & $5.52 \pm 0.67$ & 26.6 \\
T8 & $5.0 \pm 0.46$ & 24.0 \\
T9 & $4.70 \pm 0.74$ & 22.50 \\
T10 & $3.53 \pm 0.39$ & 16.65 \\
T11 & $2.93 \pm 0.26$ & 13.65 \\
\hline
\end{tabular}

Values represents mean \pm SE of five replicates of three independent experiments. T0 - Control, T1 - Half strength liquid MS medium supplemented with $0.25 \mathrm{mg} / \mathrm{L}$ IAA, T2-0.5 mg/L IAA, T3-1 mg/L IAA, T4-0.25 mg/L IBA, T5-0.5 mg/L IBA, T6-1 mg/L IBA, T7-0.25 mg/L IAA and $1 \mathrm{mg} / \mathrm{L}$ IBA, T8-0.5 mg/L IAA and $1 \mathrm{mg} / \mathrm{L}$ IBA, T9-1 mg/L IAA and IBA, T10-0.25 mg/L IBA and $1 \mathrm{mg} / \mathrm{L}$ IAA, T11-0.5 mg/L IBA and $1 \mathrm{mg} / \mathrm{L}$ IAA, IBA: Indole-3-butyric acid, IAA: Indole-acetic acid

Approximately 5-fold increase in withanolide A and withaferin A accumulation and 4-fold increase in biomass growth index (36.4 g/dl) were recorded than the control root cultures when the medium was supplemented with $4.93 \mu \mathrm{M}^{-1}$ IBA.

Auxins when supplemented in combinations with IBA and IAA, suppress the biomass growth and secondary metabolites production [12]. From the result, it was concluded that the increasing concentration of IBA alone was highly influence for lateral root formation and withanolide production. IBA is an important factor for lateral rooting and gives a better performance than IAA due to higher stability, differences in metabolism, and transport [32]. There is now a great deal of evidence 
that IBA occurs naturally in plants [17] and also suggests that IBA was the most potent auxin for the development of adventitious roots for enhanced production of secondary metabolites from $W$. somnifera. Though production of secondary metabolites in adventitious root metabolites was reported to be de novo synthesized with in root tissue. Hence, studies were conducted on tissue-specific synthesis of withanolide under in vitro condition [33]

\section{CONCLUSION}

Our results demonstrated that influence of auxins (IBA and IAA) on the lateral adventitious root growth and withanolide production of $W$. coagulans root suspension culture was reported for the first time. This may be a valuable alternative approach for producing therapeutically important bioactive principle compounds under in vitro condition. The above results are useful for the large-scale cultivation of $W$. coagulans root suspension culture for the production of withanolides.

\section{REFERENCES}

1. Jain R, Kachhwaha S, Kothari L. Phytochemistry, pharmacology and biotechnology of Withania somnifera and Withania coagulans: A review. J Med Plants Res 2012;6(41):5388-99.

2. Javanshir K. Vegetation of Bashagerd. Tehran, Iran: University of Tehran Publication; 2000. p. 156-62.

3. Panwar J, Tarafdar JC. Distribution of three endangered medicinal plant species and their colonization with Arbuscular mycorrhizal fungi. J Arid Environ 2006;65(3):337-50.

4. Ali N, Ahmad B, Bashir S, Shah J, Azam S, Ahmad M. Calcium channel blocking activities of Withania coagulans. Afr J Pharm Pharmacol 2009;3(9):439-42

5. Hemalatha S, Kumar R, Kumar M. Withania coagulans dunal - A review. Pharmacogn Rev 2008;12(2):351-8

6. Pankajavalli T, Kalaiselvi S, Oh TJ, Choi HK. Comparative chemometric profiles between leaf tissues of Withania somnifera cultured in vitro and field. IJPPS 2015;7(11):66-71.

7. Glotter E. Withanolides and related ergostane-type steroids. Nat Prod Rep 1991;8(4):415-40.

8. Misico RI, Song LL, Veleiro AS, Cirigliano AM, Tettamanzi MC, Burton $\mathrm{G}$, et al. Induction of quinone reductase by withanolides. J Nat Prod 2002;65(5):677-80.

9. Valizadeh J, Valizadeh M. Development of efficient micropropagation protocol for Withania coagulans (stocks) dunal. Afr J Biotechnol 2011;10(39):7611-6.

10. Sangwan RS, Chaurasiya ND, Lal P, Misra L, Uniyal GC, Tuli R, et al. Withanolide A biogeneration in in vitro shoot cultures of Ashwagandha (Withania somnifera DUNAL), a main medicinal plant in Ayurveda. Chem Pharm Bull (Tokyo). 2007;55(9):1371-5.

11. Mirjalili HM, Fakhr-Tabatabaei SM, Bonfill M, Alizadeh H, Cusido RM, Ghassempour A, et al. Morphology and withanolide production of Withania coagulans hairy root cultures. Eng Life Sci 2009;9(3):197-204.

12. Pankajavalli T, Kalaiselvi R, Pradeepa D, Kalaiselvi S. Effect of exogenous indole-3-butyric acid and indole-3-acetic acid on biomass and legendary with anolides from in vitro root cultures of Withania somnifera - Jawahar 20 cultivar. IJPBS 2014;5:971-9.

13. Zimmerman PM, Wilcoxon F. Several chemical growth substances which cause Initiation of roots and other responses in plants. Contrib Boyce Thompson Inst 1935;7:209-28.

14. Davies FT, Davis TD, Kester DE. Commercial importance of adventitious rooting to Horticulture. In: Davis TD, Haissig BE, editors. Biology of Adventitious Root Formation. New York: Plenum Press; 1994. p. 53-9.

15. Kovar JL, Kuchebuch RO. Commercial importance of adventitious root to agronomy. In: Davis TD, Haissig BE, editors. Biology of Adventitious Rooting Formation. New York: Plenum Press; 1994. p. 25-34.

16. Nandhagopal S, Kumari BD. Effectiveness of auxin induced in vitro root culture in chicory. J Cent Eur Agric 2007;8:73-80.

17. Praveen N, Murthy HN. Production of withanolide A from adventitious root culture of Withania somnifera. Acta Physiol Plant 2010;32:1017-22.

18. Narayan MS, Venkataraman LV. Effect of sugar and nitrogen on the production of anthocyanin in cultured carrot (Daucus carota) cells. J Food Sci 2002;67:84-6.

19. Murashige T, Skoog F. A revised medium for rapid growth and bioassays with tobacco tissue cultures. Physiol Planta 1962;15:472-97.

20. Gupta GL, Rana AC. Protective effect of Withania somnifera dunal root extract against protracted social isolation induced behavior in rats. Indian J Physiol Pharmacol 2007;51(4):345-53.

21. Jirge SS, Tatke PA, Gabhe SY. Development and validation of a novel method for simultaneous estimation of betasitosterol glucoside and withaferin A. Pharm Sci 2011;3(2):227-30.

22. Morgan PW. Agricultural Uses of Plant Growth Substances an Analysis of Present Status and Future Potential. Plant Growth Regulator Working Group, Proceeding. Vol. 6; 1979. p. 1.

23. Went FW. On a substance causing root formation. Proc K Akad Wet 1929;32:35-9.

24. Thimann KV, Went FW. On the chemical nature of the root-forming hormone of plants. Proc K Akad Wet 1934;37:456-8.

25. Overvoorde P, Fukaki H, Beeckman T. Auxin control of root development. Cold Spring Harb Perspect Biol 2010;2(6):a001537.25.

26. Beeckman T, Burssens S, Inzé D. The peri-cell-cycle in Arabidopsis. J Exp Bot 2001;52:403-11

27. Malamy JE. Lateral root formation. In: Beeckman T, editor. Root Development, Annual Plant Reviews. Vol. 37. Chichester, UK: Blackwell Publishing; 2009. p. 83-126.

28. Péret B, De Rybel B, Casimiro I, Benková E, Swarup R, Laplaze L, et al. Arabidopsis lateral root development: An emerging story. Trends Plant Sci 2009;14(7):399-408.

29. Vanneste S, Friml J. Plant signaling: Deconstructing auxin sensing. Nat Chem Biol 2012;8(5):415-6.29.

30. Delker C, Raschke A, Quint M. Auxin dynamics: The dazzling complexity of a small molecule's message. Planta 2008;227(5):929-41.

31. Kreps JA, Town CD. Isolation and characterization of a mutant of Arabidopsis thaliana resistant to alpha-methyltryptophan. Plant Physiol 1992;99(1):269-75.

32. Ludwig-Müller J, Vertocnik A, Town CD. Analysis of indole-3butyric acid-induced adventitious root formation on Arabidopsis stem segments. J Exp Bot 2005;56(418):2095-105.

33. Pankajavalli T, Kalaiselvi S. In vitro and OMICS technologies opens a new avenue for deciphering withanolide metabolism in Withania somnifera. IJPPS 2016;8(7):1-10. 